\title{
DESINGULARIZING MAPS OF CORANK ONE
}

\author{
S. J. BLANK AND C. CURLEY
}

\begin{abstract}
In 1960, A. Haefliger presented necessary and sufficient conditions for factoring a smooth map from a surface into the plane through an immersion into $R^{3}$. Here, necessary and sufficient conditions are given for factoring a map between manifolds of dimension $n, n>2$, through an immersion into a line bundle over the range. In addition, conditions are given for factoring such a map through a submersion from a line bundle over the domain.
\end{abstract}

By a desingularization of a smooth map $f: M \rightarrow V$ between manifolds we mean one of the following.

(1) An immersion $F: M \rightarrow E$ for some line bundle $\pi: E \rightarrow V$ such that $f=\pi \circ F$ or

(2) a submersion $F^{\prime}: E^{\prime} \rightarrow V$ for some line bundle $\pi^{\prime}: E^{\prime} \rightarrow M$ with zero section $s$ such that $f=F^{\prime} \circ s$.

We say $f$ desingularizes in the first (resp. second) sense with respect to $E$ (resp. $\left.E^{\prime}\right)$. If $f$ has a desingularization in the first sense, then the pull-back of the normal bundle to the image of $F$ provides a line bundle with respect to which $f$ has a desingularization in the second sense. The converse is not true. The map for Figure 1 desingularizes in the second sense but not the first. In this paper we present necessary and sufficient conditions for desingularizing a generic map with respect to a given bundle. In [1] A. Haefliger provides necessary and sufficient conditions for factoring a generic map from a closed surface into the plane through an immersion into $R^{3}$. Let $f: M \rightarrow V$ be a smooth map where $M$ is a closed smooth $n$-manifold and $V$ is a smooth $n$-manifold and let $\pi: E \rightarrow V$ be a line bundle. If there is an immersion $F: M \rightarrow E$ with $f=\pi \circ F$ we say $f$ factors through an immersion into $E$. If $f$ does factor then rank $d f>n-1$ everywhere, or equivalently, the corank of $f$ does not exceed one. The singularities of a generic $f$, by which we mean 2-generic and finite-to-one [2], with rank $d f>n-1$ everywhere, are stratified by the manifolds

$$
S_{1}=\left\{x \in M \mid \operatorname{rank} d f_{x}=n-1\right\}, \quad S_{1,1}=\left\{x \in S_{1} \mid \operatorname{rank} d\left(f_{\mid S_{1}}\right)_{x}=n-2\right\} .
$$

The map $f$ being 2-generic ensures that $S_{1}$ and $S_{1,1}$ are submanifolds of $M$.

If we regard the Klein bottle as $\left\{(\phi, \theta) \mid \phi \in S^{1}, \theta \in[0,2 \pi]\right\}$ mod the relation $(\phi, 0)=(-\phi, 2 \pi)$ then $f(\phi, \theta)=(\cos \phi+2, \theta)$ is a map from the Klein bottle to the plane (in polar coordinates). We will show that this map desingularizes in the second sense but not in the first sense. Figure 1 represents the image of this map.

Received by the editors June 21, 1979 and, in revised form, September 29, 1979.

AMS (MOS) subject classifications (1970). Primary 57D35, 57D40; Secondary 57D20, 57D45.

Key words and phrases. Immersion, submersion, line bundle, 2-generic, corank, Stiefel-Whitney class. 


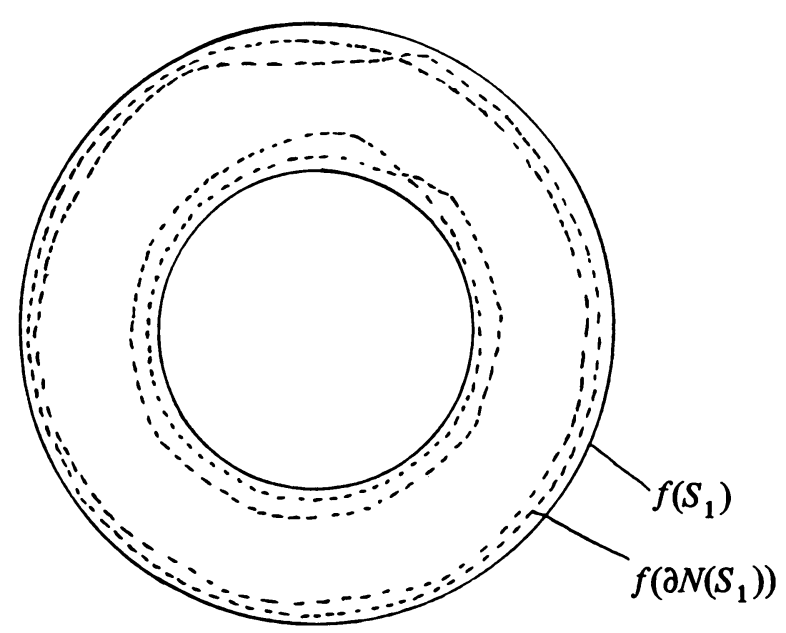

FIGURE 1: Image of $f:$ (Klein bottle) $\rightarrow \mathbf{R}^{2}$

For $n=2$ a point in $S_{1,1}$ is called a cusp. In this setting Haefliger's theorem is: $A$ generic map $f: M^{2} \rightarrow R^{2}$ factors through an immersion into $R^{3}$ if and only if each connected component of $S_{1}$ with an even (resp. odd) number of cusps has an orientable (resp. nonorientable) neighborhood.

The following example of a map $f: S^{2} \rightarrow R^{2}$ which cannot be desingularized is due to A. Haefliger:

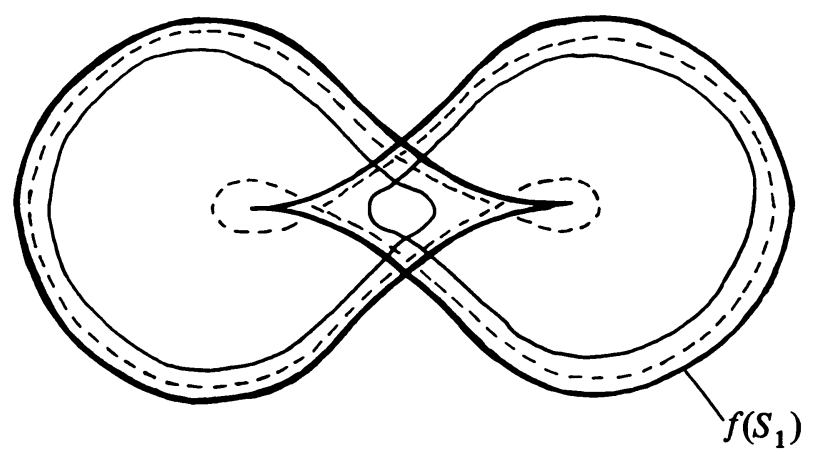

FIGURE 2

The set $S_{1}$ consists of two parallel curves $C^{\prime}$ and $C^{\prime \prime}$ each containing exactly one cusp; the image of the annulus bounded by two parallel curves between $C^{\prime}$ and $C^{\prime \prime}$ is a ribbon forming a figure eight bounded by the dashed curves; the two thin curves represent the images of the boundaries of polar caps.

For $n>2$ there is the following theorem due to Y. Saito [3]: $A$ generic map $f$ : $M^{n} \rightarrow R^{n}$ factors through an immersion into $R^{n+1}$ if (i) $M$ is orientable, (ii) rank $d f>n-1$ everywhere and (iii) $S_{1,1}=\varnothing$.

Let $N \rightarrow S_{1}$ be a tubular neighborhood of $S_{1}$ and let $K \rightarrow S_{1}$ be the line bundle 
given by the kernel of $d f$ if rank $d f \geq n-1$ everywhere. We denote the first Stiefel-Whitney classes of these bundles by $w(N)$ and $w(K)$ respectively. Similarly we denote the class of the bundle $E \rightarrow V$ by $w(E)$. Let $\left[S_{1,1}\right]$ be the class in $H^{1}\left(S_{1} ; Z_{2}\right)$ dual to the homology class represented by $S_{1,1}$ and let $i: S_{1} \rightarrow M$ be the inclusion map.

TheOREM 1. A generic map $f: M \rightarrow V$ factors through an immersion into $E$ if and only if the following conditions hold.

(i) rank $d f>n-1$ everywhere and

(ii) $\left[S_{1,1}\right]+w(N)+i^{*} f^{*} w(E)=0$.

Remark. If $M$ is orientable and $E$ is trivial, then condition (ii) is equivalent to the statement that $S_{1,1}$ bounds in $S_{1}$.

Proof of Theorem 1. Even if $f$ does not factor, we have $\left[S_{1,1}\right]+w(N)+w(K)$ $=0$.

We present an elementary proof of this fact. Let $\alpha: K \rightarrow N$ be the restriction to $K$ of the projection map from the tangent space onto the normal bundle and let $\sigma$ : $S_{1} \rightarrow K$ be a section transverse to the zero section of $K$. Since $f$ is 2 -generic the section $\alpha \circ \sigma$ is transverse to the zero section of $N$. The homology class dual to $w(K)$ is represented by the intersection of $\alpha$ and the zero section. Similarly we can represent the dual of $w(N)$ by the intersection of $\alpha \circ \sigma$ and the zero section in $N$. Since $\alpha \circ \sigma(x)$ is in the zero section if and only if either $x \in S_{1,1}$ or $\sigma(x)$ is in the zero section, these homology classes are equal. Hence $\left[S_{1,1}\right]+w(K)=w(N)$.

If $f$ factors through an immersion $F$, then $d F$ defines a bundle map from $K$ to $E$ over $f \circ i$, which is an isomorphism on the fibres. Hence $i^{*} f^{*} w(E)=w(K)$.

Conversely, if $i^{*} f^{*} w(E)=w(K)$ there is a bundle map $L: K \rightarrow E$ over $f \circ i$ which is an isomorphism on the fibres. We will use this map in factoring $f$ locally. For each $p \in M$ choose a closed disk $B_{p}$ containing $p$ such that the bundles $K$ and $E$ are trivial over $B_{p} \cap S_{1}$ and $f\left(B_{p}\right)$ respectively. Furthermore, since $f$ is finite-toone we can choose these disks so that $f(p) \notin f\left(\partial B_{p}\right)$. From this first condition we obtain, by integrating a vector field extending $K$, an immersion $F_{p}: B_{p} \rightarrow E$ for each $p$. Furthermore we require these immersions to satisfy $f=\pi \circ F_{p}$ and $d\left(F_{p}\right)_{x}(k)=\lambda_{p, x} L(k)$ where $x \in S_{1} \cap B_{p}, k \in K$ and $\lambda_{p, x}>0$. Here we identify a vector $v$ from the tangent space of $E$ with a vector in $E$ if $d \pi(v)=0$. From the second condition and the compactness of $M$ we can cover $M$ with finitely many open disks $D_{1}, D_{2}, \ldots, D_{r}$ such that $\bar{D}_{j} \subset B_{p_{j}}$ and $f\left(\bar{D}_{j}\right) \cap f\left(\partial B_{p_{j}}\right)=\varnothing$ for each $j$ and corresponding $p_{j}$.

Next choose smooth maps $\phi_{j}: V \rightarrow R^{1}$ such that (i) $\phi_{j}>0$, (ii) $\phi_{j} \circ f \equiv 0$ near $\partial B_{p_{j}}$ and (iii) $\phi_{j} \circ f>0$ on $D_{j}$. For each $j$ let $\sigma_{j}: M \rightarrow R^{1}$ be the map defined by $\sigma_{j}(x)=\phi_{j} \circ f(x)$ for $x \in B_{p_{j}}$ and $\sigma_{j}(x)=0$ otherwise. Let $\sigma_{j} F_{p_{j}}: M \rightarrow E$ be the fibre product of $\sigma_{j}$ and $F_{p_{j}}$ and let $F=\sum_{j=1}^{r} \sigma_{j} F_{p_{j}}$ be the fibre sum. Note that ker $d F \subset$ $\operatorname{ker} d(\pi \circ F)=\operatorname{ker} d f$. Since $d f$ is 1-1 away from $S_{1}$, so is $d F$. Since $\operatorname{dim} \operatorname{ker} d f_{x}=1$ for $x \in S_{1}$ it suffices to show $d F_{x}(k) \neq 0$ for $k$ a nonzero vector in the kernel of $d f_{x}$. We will calculate $d F$ in a neighborhood of $x$ in which each $F_{p_{j}}$ is of the form $\left(f, h_{j}\right)$ where $h_{j}$ maps into the fibre of $E$. Then 


$$
\begin{aligned}
d F_{x}(k) & =\sum_{j=1}^{r} d\left(\sigma_{j} F_{p_{j}}\right)_{x}(k)=\sum_{j=1}^{r} d\left(\sigma_{j} h_{j}\right)_{x}(k)=\sum_{j=1}^{r} \sigma_{j} d\left(h_{j}\right)_{x}(k) \\
& =\sum_{j=1}^{r} \sigma_{j} \lambda_{p, x} L(k)=\mu L(k)
\end{aligned}
$$

where $\mu>0$. Therefore $F$ is an immersion.

We now consider desingularization in the second sense. For a given line bundle $\pi^{\prime}: E^{\prime} \rightarrow M$ with zero section $s: M \rightarrow E^{\prime}$ we have

THEOREM 2. For a generic map $f: M \rightarrow V$ there is a submersion $F^{\prime}: E^{\prime} \rightarrow V$ with $f=F^{\prime} \circ s$ if and only if the following conditions hold.

(i) rank $d f \geqslant n-1$ everywhere and

(ii) $\left[S_{1,1}\right]=i^{*} w\left(E^{\prime}\right)$.

Proof of Theorem 2. At a point $p \in S_{1}-S_{1,1}$ the map $f$ is a fold, i.e. $f$ can be written as $f(x, y)=\left(x^{2}, y\right)$ for $x \in R^{1}$ and $y \in R^{n-1}$. If we have a submersion $F^{\prime}$ there is a unique positive direction on the fibre $E_{p^{\prime}}^{\prime}, p \in S_{1}-S_{1,1}$ determined by: $v$ is positive if and only if for any neighborhood of $p, N_{p}$, we have $F(\alpha v) \in f\left(N_{p}\right)$ for all sufficiently small $\alpha>0$. Since $f$ is 2-generic we can choose a section of $E^{\prime}{ }_{\mid s_{1}}$ which is positive on $S_{1}-S_{1,1}$ and which meets the zero section transversally with intersection $S_{1,1}$. Hence $\left[S_{1,1}\right]$ is the Stiefel-Whitney class of $E_{\mid S_{1}}^{\prime}$ as is $i^{*} w\left(E^{\prime}\right)$.

Conversely if $\left[S_{1,1}\right]=i^{*} w\left(E^{\prime}\right)$ consider the line bundle over $S_{1}$ whose fibres are the lines normal to the image $f\left(S_{1}\right)$. For a 2-generic $f$ this latter bundle is well defined and has Stiefel-Whitney class $\left[S_{1,1}\right]$. Therefore this bundle is equivalent to $E_{\mid S_{1}}^{\prime}$. Using a bundle isomorphism we can define $F^{\prime}$ on $E_{\mid S_{1}}^{\prime}$. We then extend $F^{\prime}$ so that it is constant on the fibres outside of a neighborhood of $S_{1}$.

Since any class in $H^{1}\left(M ; Z_{2}\right)$ is the Stiefel-Whitney class of some line bundle over $M$, the following are immediate for a map $f$ with rank $d f>n-1$ everywhere.

Corollary 1. A generic $f: M \rightarrow V$ desingularizes in the first sense if and only if there is a class $w \in H^{1}\left(V ; Z_{2}\right)$ which satisfies the equation $i^{*} f^{*}(w)+\left[S_{1,1}\right]+w(N)$ $=0$.

Corollary 2. A generic $f: M \rightarrow V$ desingularizes in the second sense if and only if there is a class $w \in H^{1}\left(M ; Z_{2}\right)$ which satisfies the equation $i^{*}(w)=\left[S_{1,1}\right]$.

With these corollaries we can establish the desingularization statements for the map of Figure 1.

\section{REFERENCES}

1. A. Haefliger, Quelques remarques sur les applications différentiables d'une surface dans le plan, Ann. Inst. Fourier (Grenoble) 10 (1960), 47-60.

2. M. Golubitsky and V. Guillemin, Stable mappings and their singularities, Springer-Verlag, New York, 1973.

3. Y. Saito, On decomposable mappings of manifolds, J. Math. Kyoto Univ. 1 (1961/62), 425-455.

Department of Mathematics, Northeastern University, Boston, MassachusetTs 02115

Department of Mathematics, Boston College, Chestnut Hill, Massachusetts 02167 